Trauma Berufskrankh 2014 • 16[Suppl 3]:300-304 DOI 10.1007/s10039-014-2108-0

Online publiziert: 5. Juli 2014

(c) Springer-Verlag Berlin Heidelberg 2014
G. Hübner · A. Rahmanian-Schwarz

Klinik für Plastische und Handchirurgie, Ästhetische und Rekonstruktive Chirurgie, Wuppertal
Infektionen an der Hand sind mit der häufigste Grund für eine Vorstellung in der Notfallambulanz. Eine lokale Infektion, bedingt durch Fremdkörper oder Bissverletzungen, kann sich aufgrund der komplexen Anatomie der Hand schnell auf die gesamte Hand bis in den Unterarm ausbreiten. Fehlende typische Entzündungszeichen wie Rötung, Schwellung und Überwärmung erschweren die korrekte Diagnosestellung und Therapie [5]. In der überwiegenden Zahl der Fälle ist das großzügige chirurgische Débridement indiziert, welches oft zu Weichteildefekten führt.

\section{Infektionen der Hand}

Die häufigsten Infektionen an der Hand finden sich am Nagelorgan (23,3\%; - Abb. 1) und an der Fingerpulpa (24\%). Tiefe Infektionen der Hohlhand (0,6\%), Sehnenscheidenphlegmonen $(0,3 \%)$ und Gelenkinfekte $(0,7 \%)$ sind primär seltener anzutreffen [14]. Da es aufgrund der derben palmaren Haut selten zu einer spontanen Abszessperforation kommt und sich der Infekt entlang der Bindegewebssepten in die Tiefe ausdehnen kann, ist eine schnelle Ausbreitung möglich. Zusätzlich existieren an der Hand präformierte Räume, die vom Finger bis in den Unterarm ziehen und somit eine rasche konsequente chirurgische Intervention nötig machen [12].

\section{Therapie \\ Infektion}

Bei fast allen tieferen Infektionen an der Hand ist eine chirurgische Intervention mit radikalem Débridement (『 Abb. 2), Spülung und Drainage sowie kalkulierter und sekundär erregergerechter Antibiotikatherapie mit Ruhigstellung der Extremität notwendig [4].

Insbesondere bei ausgedehnten Infekten wie Hohlhandphlegmonen, septischen Arthritiden oder nekrotisierenden Faszitiden sind mehrzeitige Débridements mit Nekrosenabtragung und offener Wundbehandlung mit Wundkonditionierung durch Unterdruckwundtherapie [NPWT („,negative-pressure wound therapy“)] unabdingbar.

\section{Defektrekonstruktion}

Der aus der chirurgischen Behandlung resultierende Haut-/Weichteildefekt muss anschließend oft mit einer plastischen Rekonstruktion gedeckt werden. Dabei darf das zur Deckung notwendige Gewebe nicht aus betroffenem oder dem Infekt angrenzendem Gewebe gewonnen werden. Die plastische Rekonstruktion erfolgt daher erst nach Ausheilung des Infekts im gut durchbluteten Gewebe [7].

Die Art und Technik der Rekonstruktion hängen von der Lokalisation und dem Ausmaß des Weichgewebedefekts ab. Die Defektdeckung erfolgt entlang der sog. rekonstruktiven Leiter, von der Hauttransplantation über lokale und regiona- le Lappenplastiken bis zum freien Gewebetransfer.

\section{Weichteildefekte am Finger}

Weichteildefekte an der Fingerkuppe bis zur Mittelphalanx können oft mit lokalen Lappenplastiken gedeckt werden. Ein einfacher und sicherer Lappen ist die VYPlastik, die insbesondere bei Defekten an der Fingerkuppe genutzt werden kann.

Regionale Lappen können bei dieser Lokalisation als Cross-Finger-Lappenplastik [8] bzw. bei dorsalem Weichteilschaden mit einem reversen Cross-Finger-Lappen (• Abb. 3) versorgt werden. Sollen sensible Funktionen wiederhergestellt werden, kann ein neurovaskulärer Insellappen verwendet werden. Dies ist insbesondere zur Gewährleistung der sensomotorisch kontrollierten Greiffunktion bei Defekten an der ulnaren Daumenoder radialen Zeigefingerseite notwendig.

Eine andere Möglichkeit zur Defektdeckung an der Fingerkuppe stellt der Thenarlappen dar.

Stehen keine Möglichkeiten zur Deckung mittels regionalem Lappen zur Verfügung, können als Fernlappen ein Leistenlappen (- Abb.4) oder aber, bei adäquatem Wundgrund, die Transplantation von Vollhaut zum Einsatz kommen.

Das proximale Interphalangealgelenk stellt aufgrund seiner wichtigen Funktion bei der Fingerbeweglichkeit eine Herausforderung für die Deckung dar. Eine vollständige Beweglichkeit des Gelenks muss gewährleistet sein. Transpositionslappen, wie der Bunnell-Lappen, können als lokale Lappenplastiken angewandt werden. 


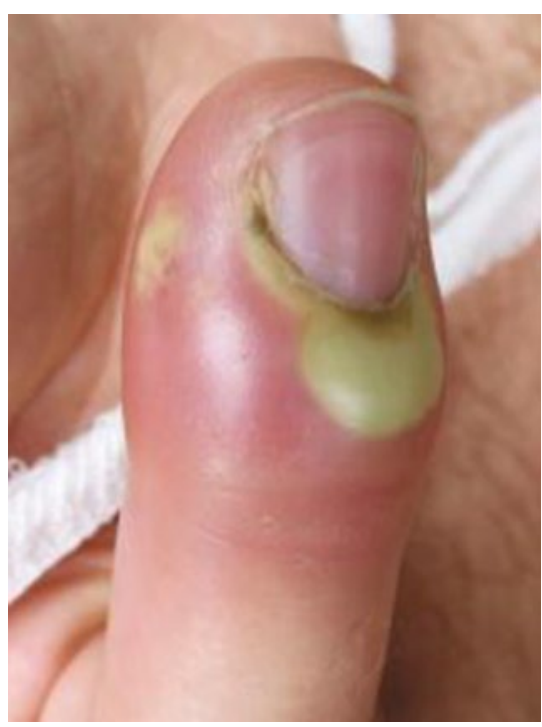

Abb. $1 \Delta$ Nagelwallentzündung mit Eiteransammlung

Im Bereich der regionalen Lappen bieten sich Cross-Finger-Lappen als einfache und schnelle Lösung an. Palmare Defekte werden mit Advancement- oder homodigitalen Insellappen gedeckt. Eine weitere Möglichkeit stellt der venöse Durchstromlappen dar.

Der dorsale Metakarpalarterienlappen bietet breite Anwendungsmöglichkeiten, die zur Defektdeckung vom Metakarpus bis zum dorsalen PIP-Gelenk (PIP: „proximal interphalangeal joint"), als modifizierte Variante auch bis zum Mittelglied, reichen.

Am Metakarpophalangealgelenk wird neben den bereits beschrieben Techniken auch der Kite-Lappen genutzt.

\section{Haut-Weichteil-Defekte der Hand}

Defekte am Metakarpus sind eine Domäne der regionalen Lappen oder Fernlappen. Dorsalseitig können auch lokale Lappenplastiken wie der Limberg-, der Rotations- oder der Advancement-Lappen Verwendung finden.

Der Interosseus-posterior-Lappen (• Abb.5) ist der am häufigsten verwendete distal gestielte Lappen, um Weichteildefekte am Metakarpus zu decken. Lange Zeit wurde an dieser Lokalisation auch der Radialislappen ( $\bullet$ Abb. 6, [10]) benutzt. Aufgrund des kosmetisch störenden Hebedefekts liegen die Schwerpunkte heute jedoch auf anderen Möglichkeiten. So wurden in den letzten Jahrzehnten

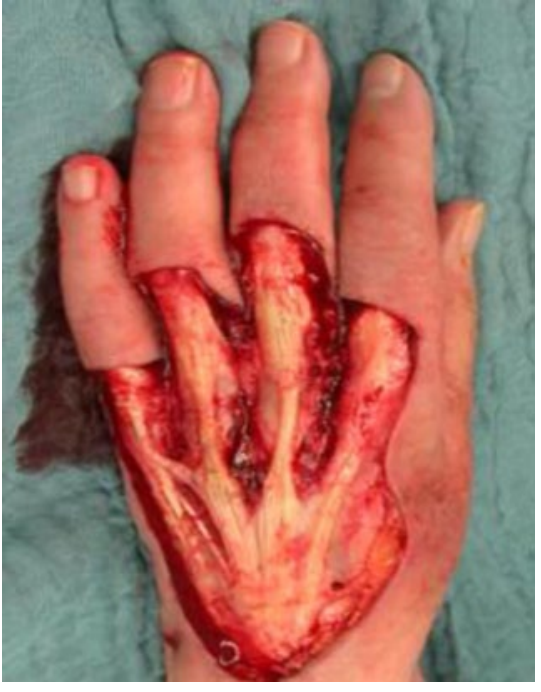

Abb. $2 \triangle$ Massive Infektion der Hand nach Débridement

die Techniken des freien Gewebetransfers verfeinert, und dieser stellt heute eine sichere Lappenplastik dar. Insbesondere bei größeren Gewebedefekten können der laterale Oberarmlappen, ein Paraskapularlappen oder der ALT-Lappen (ALT: , antero lateral tight") verwendet werden. Ein Nachteil sind die großen Volumina dieser Lappen, die oft einer Korrektur in weiteren Eingriffen bedürfen. Bei der Rekonstruktion mit Hilfe einer Muskellappenplastik (z. B. M.-serratus-anterior-Lappenplastik) erhält man ein ausgezeichnetes Transplantatlager für weitere Knochen-, Nerven- und/oder Sehnentransplantationen [3]. Die Dicke der freien mikrovaskulären Muskellappenplastik erreicht nach sekundärer Atrophie des denervierten Muskels eine akzeptable Kontur.

Bei Defekten im Hohlhandbereich sollte auf vom Unterarm gestielte lokale Lappenplastiken aufgrund deren dicker Subkutanschicht verzichtet werden. Häufig kommt es hierbei zur sog. Savonnage, d. h. einer starken Verschieblichkeit des Hautmantels.

Anforderungen an die Hautdeckung im Bereich des Handrückens ist, aus funktioneller Sicht, die Gewährleistung eines ausreichenden Gleitlagers für die Strecksehnen [16]. Funktionell muss beachtet werden, dass es von der proximalen dorsalen Handgelenkfalte bis zu den PIP-Gelenken zu großen Hautverschiebungen bei Hand- und Fingerbewegungen kommt.
Trauma Berufskrankh 2014 - 16[Suppl 3]:300-304

DOI 10.1007/s10039-014-2108-0

c) Springer-Verlag Berlin Heidelberg 2014

\section{G. Hübner · A. Rahmanian-Schwarz \\ Plastische \\ Rekonstruktionsmöglichkeiten nach Infektionen an der Hand}

\section{Zusammenfassung}

Hintergrund. Eine lokale Infektion an der Hand kann sich aufgrund deren komplexer Anatomie schnell auf die gesamte Hand bis in den Unterarm ausbreiten.

Therapie. Fast alle tieferen Infektionen an der Hand erfordern eine chirurgische Intervention mit radikalem Débridement, Spülung und Drainage sowie kalkulierter und sekundär erregeradaptierter Antibiotikatherapie mit Ruhigstellung. Der resultierende Haut-/ Weichteildefekt muss häufig mittels plastischer Rekonstruktion gedeckt werden. Deren Art und Technik hängen von der Lokalisation und dem Ausmaß des Defekts ab. Als Richtlinie dient die sog. rekonstruktive Leiter, von der Hauttransplantation über lokale und regionale Lappenplastiken bis zum freien $\mathrm{Ge}-$ webetransfer. Sehr ausgedehnte, auch Knochen und Gelenke betreffende Defekte erfordern individuelle Rekonstruktionstechniken.

\section{Schlüsselwörter}

Hand · Infektion · Radikales Débridement . Weichteildefekt - Defektrekonstruktion

\section{Plastic reconstruction options after hand infections}

\section{Abstract}

Background. Due to the complex anatomy a local infection of the hand can rapidly spread to the whole hand and even to the forearm. Therapy. Nearly all deeper infections of the hand require surgical intervention with radical débridement, flushing and drainage as well as calculated and secondary pathogenoriented antibiotic therapy with immobilization. The resulting skin and soft tissue defect must often be covered by plastic reconstruction. The type and technique used depend on the localization and extent of the defect. The so-called reconstructive ladder serves as a guideline for skin transplantation, local and regional flap plastic surgery up to free tissue transfer. Very extensive defects as well as those affecting bone and joints necessitate individualized reconstruction techniques.

\section{Keywords}

Hand · Infection - Débridement · Soft tissue injuries - Reconstructive surgical procedures 


\section{Hand- und Unterarminfektionen}
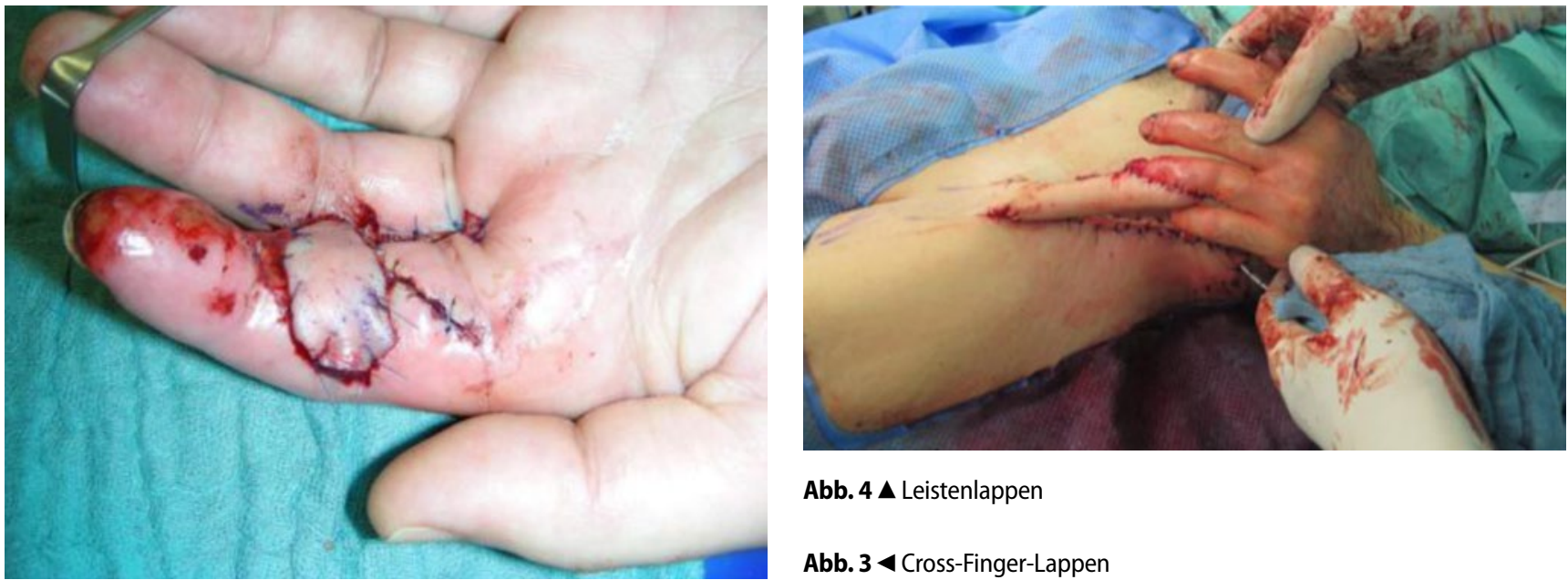

Abb. 4 \ Leistenlappen

Abb. $3<$ Cross-Finger-Lappen
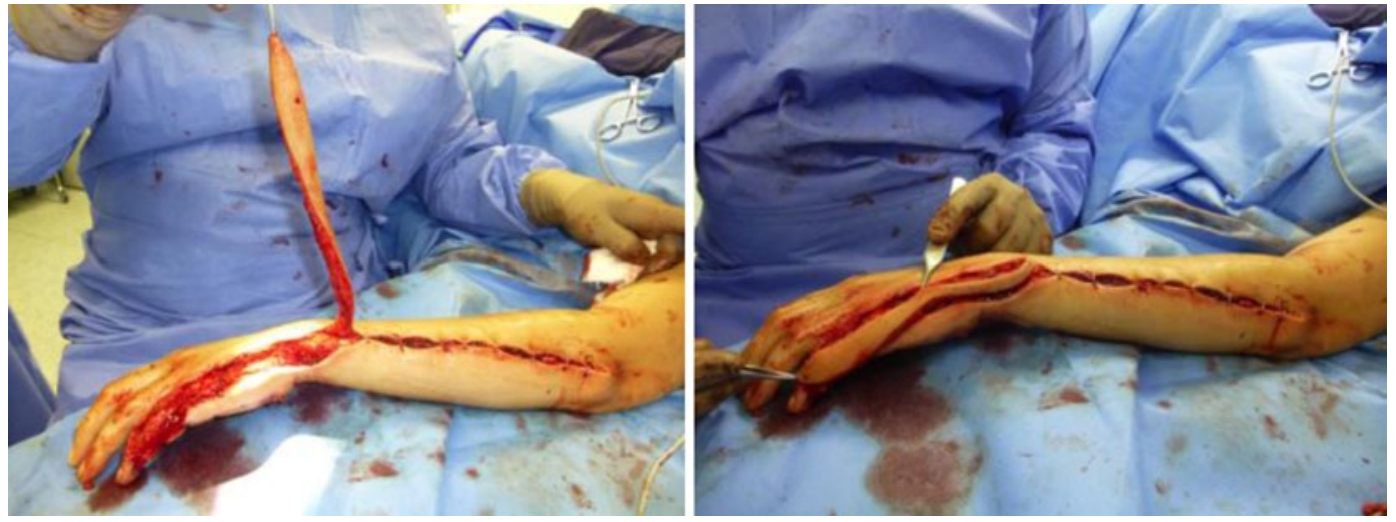

Abb. $5<$ Interosseus-posterior-Lappen
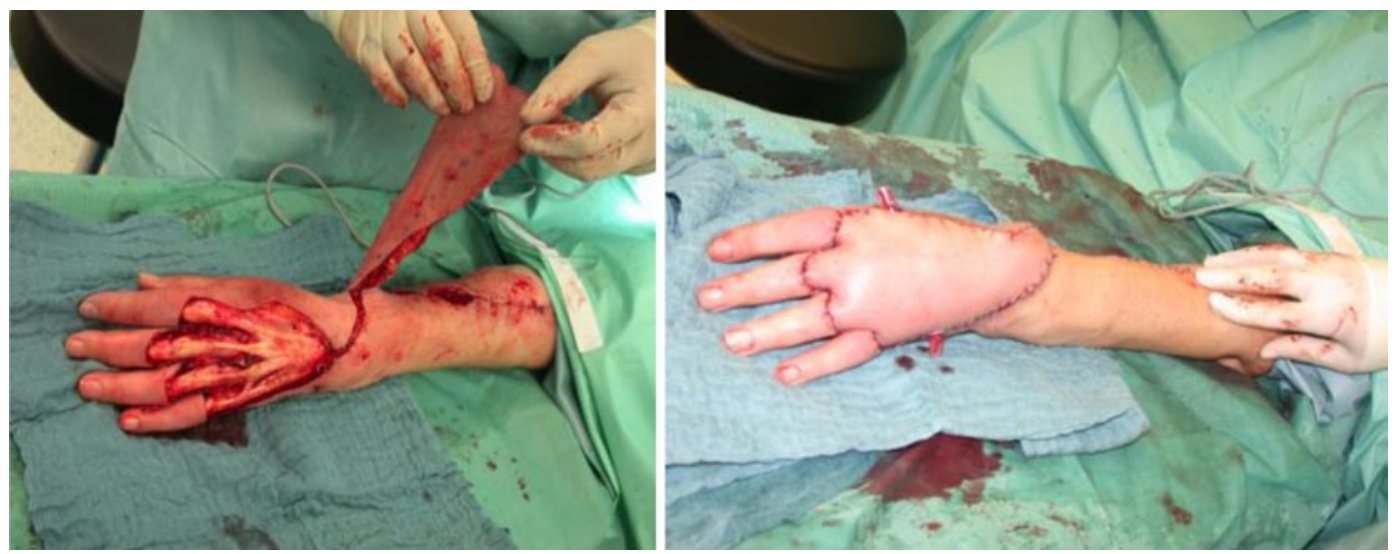

Abb. $6 \varangle$ Radialislappen

Daher sollte bei der Planung der Rekonstruktion ein relativer Hautüberschuss bedacht werden [1]. Bei kleinen zentralen Handrückendefekten kann oft eine lokale, defektangrenzende Lappenplastik zur Anwendung kommen. Größere Defekte mit intaktem Peritendineum des Streckapparats können mit Hilfe einer Volloder Spalthauttransplantation verschlossen werden [11]. Alternativ können lokale Lappenplastiken wie hautgestielte Insel- lappenplastiken verwendet werden. Bei größeren Defekten sollte einer freien mikrovaskulären Lappenplastik der Vorzug gegenüber einer gestielten Fernlappenplastik gegeben werden.

\section{Defektdeckung am Daumen}

Eine Besonderheit in der Defektdeckung stellt der Daumen dar. Am Daumenendglied werden eine hohe mechanische Beanspruchbarkeit, eine gute Sensibilität und die Erhaltung der maximalen Daumenlänge zur vollen Oppositionsfähigkeit angestrebt [18]. Lokale Lappenplastiken, wie eine VY-Dehnungslappenplastik nach Tranquilli-Leali, eine Dehnungslappenplastik nach Moberg oder eine Translationslappenplastik nach Hueston haben strenge Indikationen in den Zonen 1-2, um die anspruchsvollen postoperativen Ergebnisse zu erreichen. Der häufig verwendete Foucher-Lappen stellt als neuro- 

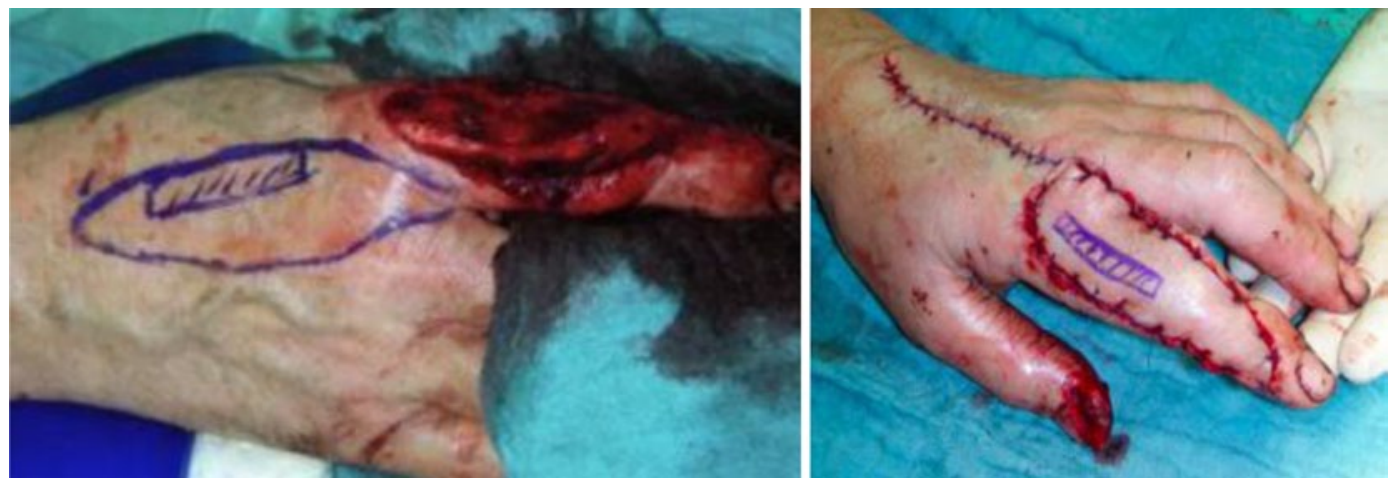

Abb. 74 Kombinationslappen - DMCA- und M.extensor-indicis-Lappen, $D M C A_{\text {„ }}$ dorsal metacarpal artery"
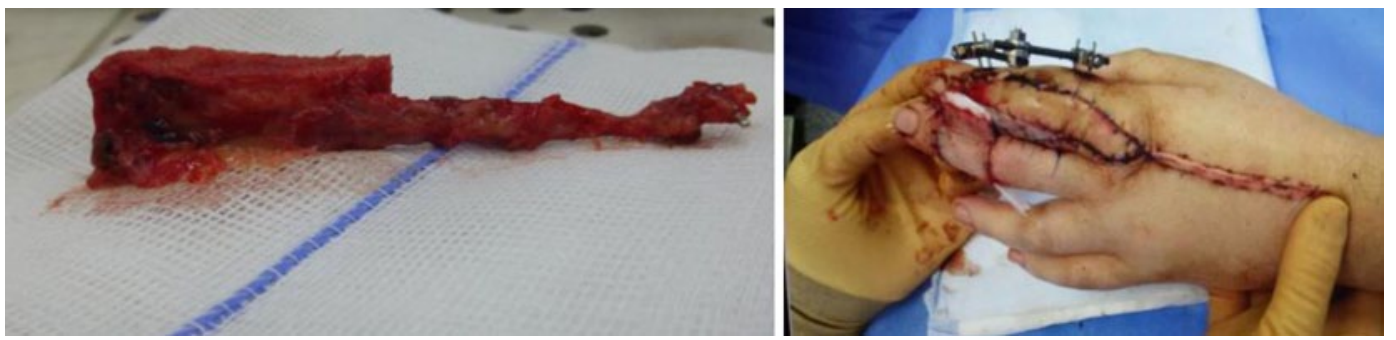

Abb. $8<$ Vaskularisiertes Knochentransplantat aus dem medialen Femurkondylus, DMCA- und CrossFinger-Lappen, $D M C A_{\text {" }}$ dorsal metacarpal artery"

vaskulärer Insellappen die sensible Versorgung des gedeckten Daumendefekts ab Zone 3 sicher. Andere Möglichkeiten sind ein homodigitaler neurovaskulärer Inseloder ein Cross-Finger-Lappen. Als weitere Therapiemöglichkeit kommen gestielte Lappenplastiken aus dem Hand- oder Unterarmbereich in Frage. Ist dies nicht möglich, ist auch hier eine Fernlappenplastik erforderlich, z. B. mittels Leistenoder Bauchhautlappenplastik. Wegen der Notwendigkeit der frühzeitigen Mobilisierung, der leichteren Pflege und einer geringeren psychischen Beeinträchtigung sind die mikrovaskulären Lappenplastiken den gestielten Fernlappenplastiken vorzuziehen [18].

\section{Komplexe Rekonstruktionen}

Sehr ausgedehnte Defekte, die auch Knochen und Gelenke mitbetreffen, bedürfen individueller Rekonstruktionstechniken. Hier können verschiedene Lappenplastiken kombiniert werden ( $\mathbf{\bullet}$ Abb. 7). Auch Lappenplastiken mit gleichzeitigem Sehnentransfer sind im Einzelfall gerechtfertigt. Bei kleineren Weichteildefekten mit partiellem oder segmentalem Knochendefekt sollte man an die Möglichkeit eines freien mikrovaskulären Knochentransfers vom vorderen Beckenkamm, von der Skapula oder aus dem Unter- und Oberarmbereich denken. Alternativ wird ein vaskularisiertes Knochentransplantat aus dem medialen Femurkondylus entnommen und in die Defektzone transplantiert (• Abb. 8). Dadurch kann gut vaskularisiertes Gewebe eingebracht werden, wodurch sich Knochen schneller konsolidieren kann und die Komplikationsrate herabgesetzt wird [9].

Septische Arthritiden mit der Folge von Fingergelenkdefekten resultieren in einer starken funktionellen Beeinträchtigung mit Schmerzen, Bewegungs- und/ oder Kraftverlust [2]. Solche Gelenkzerstörungen finden sich häufig nach den im ersten Anschein oft unterschätzten FightBite-Clenched-Fist-Verletzungen. Therapeutische Möglichkeiten bei einer Gelenkzerstörung im Fingerbereich hängen vom Ausmaß der destruierten Gelenkflächen, vom Alter des Patienten und von der funktionellen Beeinträchtigung ab. Therapieoptionen sind z. B. die Fingergelenkdenervierung [13], Perichondriumtransplantate [6], Interpositionsarthroplastiken [17], Fingergelenkprothesen [15], Gelenkversteifungen oder vaskularisierte Gelenktransplantationen aus dem Hand- oder Fußbereich.

\section{Korrespondenzadresse}

\section{Dr. G. Hübner}

Klinik für Plastische und Handchirurgie, Ästhetische und Rekonstruktive Chirurgie, Heusnerstraße 40, 42283 Wuppertal gunnar.huebner@helios-kliniken.de

\section{Einhaltung ethischer Richtlinien}

Interessenkonflikt. G. Hübner und A. RahmanianSchwarz geben an, dass kein Interessenkonflikt besteht.

Alle Patienten, die über Bildmaterial oder anderweitige Angaben innerhalb des Manuskripts zu identifizieren sind, haben hierzu ihre schriftliche Einwilligung gegeben. Im Falle von nicht mündigen Patienten liegt die Einwilligung eines Erziehungsberechtigen oder des gesetzlich bestellten Betreuers vor.

Dieser Beitrag beinhaltet keine Studien an Menschen oder Tieren.

The supplement containing this article is not sponsored by industry.

\section{Literatur}

1. Colville J (1992) The management of skin loss on the dorsum of the hand and finger. Skin cover in the injured hand. Churchill Livingstone, Philadelphia, S 150-158

2. Dautel G (1995) Reconstruction articulaire. In: Merle M, Dautel G (Hrsg) La main traumatique. Masson, Paris, S 15-54

3. Dautel G, Merle M (1992) Couverture cutanee. La main traumatique. Masson, Paris, S 74-178

4. Gerstung $\mathrm{TI}$ (2012) Infektionen der Hand. Trauma Berufskrankh [Suppl 1] 14:93-99 


\section{Hand- und Unterarminfektionen}

5. Hanebuth G (2013) Therapie von Infektionen an der Hand. Trauma Berufskrankh [Suppl 2] 15:169173

6. Johansson SH (1987) Perichondrial grafting to the finger joints. In: Urbaniak JR (Hrsg) Microsurgery for major limb reconstruction. Mosby, St. Louis, S 294-297

7. Kall S (2005) Chirurgische Therapie von Infektionen an der Hand. Chirurg 76:615-628

8. Kappel DA (1985) The cross-finger-flap: an established reconstructive procedure. Hand Clin 1:677

9. Lexer E (1924) Die freien Transplantationen. Enke, Stuttgart

10. Mühlbauer W (1982) The forearm flap. Plast Reconstr Surg 70:336

11. Partecke B-D (1997) Defekte in der Hohlhand und am Handrücken. Der Weichteilschaden an der Hand. Hippokrates, Stuttgart, S 13-63

12. Pillukat $T$ (2008) Infektionen an der Hand. Orthop Unfallchir Up2date 195-214

13. Sokolov C (1994) Traitement non prothetique des atteintes degeneratives de IPP et de la MCP. 7e Symposium de I'Institut Français de la Main, Paris

14. Stevanovic M (2005) Acute infections in the hand. In: Hotchkiss R, Pederson W, Wolfe S Green D (Hrsg) Green's operative hand surgery. Churchill Livingston, Philadelphia

15. Swanson AB (1972) Flexible implant resection arthroplasty. Hand 4:119-134

16. Tiedemann I (1987) Defekte an der Streckseite der Finger. Der Weichteilschaden an der Hand. Hippokrates, Stuttgart, S 87-105

17. Tupper JW (1989) The metacarpophalangeal volar plate arthroplasty. J Hand Surg Am 14:371-375

18. Wilhelm P, Hierner G (1997) Lappenplastiken in der Handchirurgie. Urban \& Schwarzenberg, München Wien Baltimore 